\title{
Guest Editorial: Special Issue on Artificial Intelligence, Machine Learning, Data Science, and Libraries
}

The Artificial Intelligence (AI) and Machine Learning (ML) movement has nowadays touched almost every aspect of our lives. The prevalence of the AI movement can be attributed to the neverending creation of new data, the availability of data at our fingertips, and the increased computing power to process that data. In the last decade, we have seen more and more data-based AI applications emerge in sectors ranging from consumer goods (e.g. Google's Alexa, self-driving cars) to health care (e.g. DeepMind) to social media. However, this is likely only the beginning, as we are rapidly entering an AI-based society that will change how we live and interact with each other and the world around us.

What is Artificial Intelligence and Machine Learning?

In a whitepaper published by Ex Libris titled Artificial Intelligence in the Library: Advantages, Challenges and Tradition, ML is defined to be “... when machines create their own classifications by learning from examples, dramatically accelerating statistical pattern recognition. In other words, AI technologies and applications are all based on machine learning algorithms." It also quotes Catherine Nicole Coleman as saying: "The power (of AI) lies in the fact that machines can recognize patterns efficiently and routinely, at a scale and speed that humans cannot approach."

Are libraries ready to embrace AI technologies? Where do we start?

Fortunately, a few visionary leaders and pioneers among us have already started that process. Stanford University Libraries, for instance, in collaboration with the National Library of Norway and a few other institutions, hosted two conferences in last two years titled Fantastic Futures: International Conference on AI for Libraries, Archives, and Museums. In 2018, the library at the University of Rhode Island opened an AI Lab, the first of its kind in the library world. Furthermore, the Library Technology Reports, an American Library Association (ALA) publication, published an issue titled "Artificial Intelligence and Machine Learning in Libraries" in 2019. In the foreseeable future, we will definitely see more events, projects, and publications with regard to AI, ML, and DS in the field of library and information science.

I am excited to report that, after more than six months of preparation and hard work, our journal's special issue on AI, ML, Data Science (DS), and Libraries is published today. What follows is a brief introduction to each article in this issue.

In his opening remarks for the Fantastic Futures $2^{\text {nd }}$ International Conference held at Stanford University Libraries in December 2019, Aslak Sira Myhre, the director of the National Library of Norway, gave an inspirational call-to-action to libraries. The traditional role of libraries 
as the gatekeepers of human knowledge is behind us, Myhre advocated. We instead need to be the gate-openers, the enlighteners and educators, and the agents and missionaries for the knowledge in our collections. And to do that, we must use AI and ML as tools to analyze our collections that is, when put together, all of humankind knowledge, to gain insights, and to open the door for our patrons for new knowledge and wisdom. The transcript can be found in the editorial section.

In the "Featured Articles" section, we have five articles, four of them dealing with different aspects of AI, ML, and DS.

Coleman from Stanford takes on a critical issue regarding potential consequences of utilizing ML and AI in libraries without proper forethought, which introduces the inherent data bias and algorithmic bias presented in the field of AI, ML and DS. This article presents evidence without monitoring by outside forces, industries tend towards efficiency over accountability. Libraries need to play a role in the design and adoption of correct AI application by managing bias when analyzing their collections to ensure equality, accountability, and sustainability.

The next article involves utilizing a ML technique to solve a specific library problem. In this pioneering work of applying ML methods in the field of library science, Flannery from the University of Notre Dame managed to automatically generate summaries for a special library collection of more than five thousand Catholic Pamphlets by implementing summarization techniques through Natural Language Processing (NLP). His experiment opens the door for programmers in libraries around the world to learn and practice different techniques in ML and AI, such as Summarization and Classification in ML and Deep Learning and neural networks in AI.

The next two articles both deal with an important area of data science, the Digital Humanities, and specifically, the Geographical Information System (GIS). Digital Humanities, a field of study at the intersection of computer science, big data, and the humanities, poses a great opportunity for libraries to engage with the research and scholarly activities of higher education. Luo and Park (University of Windsor)'s article describes their project of developing a web-based learning module that aims to explore the possibility of using community asset mapping and GIS as an integrated tool to promote service learning in social work education. The article by Gao (University of Houston) and Wang (The College of New Jersey) presents a case study that focuses on how to provide GIS support and service to the campus community without a dedicated GIS librarian.

The last feature article, by MoChridhe from the American Theological Library Association (Atla), discusses the importance of International Covenant on Civil and Political Rights (ICCPR) to libraries by examining ICCPR Article 27's guarantees of cultural and language rights for minorities on the provision of non-English library collections and services, arguing that the treaty provides a legal foundation for library advocacy to support the work envisioned in the ALA's guideline documents.

In the "Reports from the Field" section, we include two articles.

Luo from the University of Windsor showcases a concrete example of using GIS technology to examine geographic accessibility to supermarkets and fast food outlets and how they 
interact with neighbourhood socioeconomic and zoning characteristics to identify food deserts and food swamps in Windsor, Ontario, Canada.

The last article, by Diao from the City University of New York, presents a personal account in the concept, design, implementation, and assessment of Case-Based Leaning (CBL) in an information literacy classroom. It discusses the advantages and limitations of this method, offers suggestions about the future of CBL, and points out potential concerns related to the evaluation of workload, librarians' responsibilities, and workplace culture.

\section{Yongming Wang, The College of New Jersey, USA}

\section{Reference}

Ex Libris (n.d). Artificial Intelligence in the Library: Advantages, Challenges and Tradition. An Ex Libris Whitepaper. Retrieved July 11, 2020 from https://cdn2.hubspot.net/hubfs/2909474/Ex\%20Libris\%20Artificial\%20Intelligence\%20 White\%20Paper.pdf.

\section{Resources}

1. Fantastic Futures 2019: $2^{\text {nd }}$ International Conference on AI for Libraries, Archives, and Museums. https://library.stanford.edu/projects/fantastic-futures. Accessed on July 15, 2020.

2. Library Technology Reports (Vol. 55, no. 1), "Artificial Intelligence and Machine Learning in Libraries," Edited by Jason Griffey. https://journals.ala.org/index.php/ltr/issue/view/709. Accessed on July 15, 2020.

3. AI Labs at University of Rhode Island Library. https://web.uri.edu/ai/. Accessed on July $15,2020$.

\section{Editing contributors to this issue}

Copy Editing: Maureen Gorman, Denise Lynne Montgomery, Jessica Koos, Jason Wang, Julia Zheng

Layout Editing: Cindy Li, Gordon Xu 